Mariusz Jakosz

ORCID: 0000-0001-9606-679X

Schlesische Universität, Katowice

DOI: $10.19195 / 0435-5865.144 .22$

\title{
Hochschulgermanistik in Oberschlesien - Sprachstand und Motivation der Germanistikstudierenden der Schlesischen Universität Katowice
}

\begin{abstract}
s
Das Germanistikstudium in Polen ist seit einiger Zeit von einer merklichen Krise betroffen, was mit der stark abnehmenden Zahl der Lernenden und Studierenden im Bereich des Deutschen als Fremdsprache einhergeht. Im Beitrag wird zunächst der aktuelle Status des Deutschen als Fremdsprache in Polen umrissen. Dann geht der Autor auf die gegenwärtige Situation der polnischen Germanistik am Beispiel der Schlesischen Universität Katowice ein. Die Aufmerksamkeit richtet sich auf die Analyse der Ergebnisse eines grammatisch-lexikalischen Einstufungstests, der zum Zwecke der Sprachstandsdiagnose am Institut für Germanische Philologie zu Beginn des akademischen Jahres durchgeführt wird. Darüber hinaus verweist der Autor auf die Facetten der Lernmotivation anhand ausgewählter Sprachlernbiografien von Germanistikstudierenden.
\end{abstract}

Schlüsselwörter: Germanistikstudium, Motivation, Sprachniveau, Deutsch, Sprachlernbiografie

\section{German studies at university level in Upper Silesia - students' language level and motivation, taking the University of Silesia in Katowice as an example}

One can notice quite a serious crisis that German studies in Poland have been facing for some time, which is connected with the fact that the number of those who learn German as a foreign language and the number of students of German are significantly dwindling. At the beginning of the article, the author describes the current status of German as a foreign language in Poland. Next, he discusses the present situation of academic German studies in Poland, paying particular attention to the profile of German studies offered by the University of Silesia in Katowice. The researcher concentrates 
on the results of the vocabulary and grammar test that is administered at the Institute of German Studies at the beginning of the academic year in order to diagnose students' language skills. Finally, the author shows various reasons for learning German and taking up German studies using selected language biographies of students.

Keywords: German studies, motivation, language level, German language, language biography

Mariusz Jakosz, Uniwersytet Śląski, Instytut Filologii Germańskiej, ul. gen. Stefana Grota-Roweckiego 5, 41-205 Sosnowiec, Polen, E-Mail: mariuszjakosz@interia.pl

Received: 23.09.2018, accepted: 8.04.2019

Die polnische Hochschulgermanistik erlebt seit einiger Zeit eine merkliche Krise, was mit der stark abnehmenden Zahl der Lernenden und Studierenden im Bereich des Deutschen als Fremdsprache einhergeht. Zwischen 2008 und 2013 sank die Anzahl der Germanistikstudierenden um 38 Prozent und die Anzahl der Lernenden im Rahmen des studienbegleitenden Deutschunterrichts um 36 Prozent (Földes 2016: 14-16). Laut Bericht des Auswärtigen Amtes (2015: 19) lernten bzw. studierten im Jahr 2015 Deutsch insgesamt 96.555 Personen von 1.6 Millionen Studierenden. Davon wählten die meisten (87.300 Studierende) Deutsch als studienbegleitendes Fach und 9.255 Studierende entschieden sich für das Germanistikstudium. Im akademischen Jahr 2015/2016 waren nur noch 7.726 Personen im Fach Germanistik eingeschrieben, das an 23 staatlichen Hochschulen, an 19 staatlichen Berufshochschulen und an 13 privaten Hochschulen in Polen angeboten wird (Lada 2017a: 19). Ein erneuter Anstieg der Deutschlernenden scheint in Polen aktuell nicht möglich zu sein, was auf den demographischen Wandel und die zurückgehende Stellung des Deutschen im polnischen Schulsystem zurückzuführen ist (Auswärtiges Amt 2015: 19; Janoszczyk 2016: 44-47; Mihułka 2016: 62; Sipowicz/Najbert/Radojewska 2016: 142; Łada 2017b: 5). Die Folge ist, dass viele private Hochschulen geschlossen werden müssen und es an manchen Fakultäten an Studienbewerberinnen und -bewerbern fehlt. Dies wird in den nächsten Jahren zu einem signifikanten Problem für fast alle Hochschulen und Universitäten in Polen werden (Sipowicz/Najbert/Radojewska 2016: 142; Szczęk/ Kałasznik 2016: 105; Łada 2017a: 15; Łada 2017b: 5).

Im vorliegenden Beitrag wird zunächst der aktuelle Status des Deutschen als Fremdsprache in Polen umrissen, um dann auf die gegenwärtige Situation der polnischen Germanistik unter Berücksichtigung des Profils der Schlesischen Universität Katowice einzugehen. Im Mittelpunkt stehen die Ergebnisse einer statischen Untersuchung, an der die Germanistikstudierenden in der ersten Woche des akademischen Jahres teilnahmen. Zum Zweck der Sprachstandsdiagnose wurde unter ihnen ein grammatisch-lexikalischer Einstufungstest eigens durchgeführt. Beleuchtet werden auch die Facetten der Lernmotivation am Beispiel ausgewählter 
Sprachlernbiografien von Germanistikstudierenden, die einen tieferen Einblick in ihre Entscheidungs-, Lern- und Motivationsprozesse gewährleisten. Ohne Motivation gibt es kein effizientes Lernen und somit auch keinen Lernfortschritt. Unsere Aufmerksamkeit richtet sich auf solche Faktoren, die die Lernmotivation fördern bzw. beeinträchtigen können. Dieser Beitrag erhebt keinen Anspruch darauf, ein ganzheitliches Bild des Germanistikstudiums in Polen zu zeichnen, die Ergebnisse haben nur Orientierungscharakter und können nicht als repräsentativ für andere Hochschulen in Polen gelten.

\section{Zum Stellenwert des Deutschlernens in Polen}

Deutsch nimmt als Schulfremdsprache im generellen Vergleich eine starke zweite Position nach Englisch ein: 90\% der polnischen Schülerinnen und Schüler lernen Englisch, 40\% Deutsch, 5\% Russisch und 3\% Französisch (Auswärtiges Amt 2015: 19). Wenn man den Anteil der jungen Deutschlernenden mit denjenigen, die insgesamt Fremdsprachen lernen, vergleicht, so lässt sich festhalten, dass Deutsch, statistisch gesehen, überwiegend an Schulen in den Woiwodschaften Lebuser Land, Westpommern und Niederschlesien vertreten ist, somit in den Regionen, die in der Nähe der polnisch-deutschen Grenze liegen. Der geringste Prozentanteil an Deutsch lernenden Schülerinnen und Schülern ist dagegen in den Woiwodschaften Lublin und Podlachien, d.h. an der so genannten polnischen Ostwand, zu verzeichnen (Ammon 2015: 1011-1012; Łada 2017a: 15). ${ }^{1}$ Außerhalb des Schulsystems wird Deutsch in Polen auch an Hochschulen, an den Goethe-Instituten in Warschau und Krakau, am Österreich-Institut in Warschau, Krakau und Breslau sowie an zahlreichen privaten Sprachschulen erlernt (Łada 2017a: 11).

Infolge der demografischen Veränderungen ${ }^{2}$ und des allgemeinen Rückgangs der Schülerzahlen sank die Anzahl der Deutschlernenden im Vergleich zum Jahr 2010. Im Jahr 2010 lernten noch 2.282.822 Schülerinnen und Schüler $(27,9 \%)$ Deutsch, im Jahr 2015 bereits nur 1.946.523 Schülerinnen und Schüler $(23,6 \%)$ (Łada 2017a: 14). Zu betonen ist aber, dass die Anzahl der polnischen Deutschlernenden an Schulen in den letzten fünf Jahren nur um 8\% sank, während sich die Gesamtschülerzahl in demselben Zeitraum um 25\% verringerte (Auswärtiges Amt 2015: 19).

${ }^{1}$ Von Bedeutung ist hier u.a. die Studie von Mackiewicz (2013), der sich mit dem Problem der Motivation der polnischen DaF-Lernenden aus der Sicht der regionalen Unterschiede auseinandersetzt.

2 Laut den Schätzungen wird die Einwohnerzahl Polens von 2010 bis 2035 von ca. 38 Mio. auf 36 Mio. sinken (Auswärtiges Amt 2015: 19). 
Tab. 1: Zahl der Deutschlernenden (Erwachsene, Kinder und Jugendliche) an Schulen in den Jahren 2010 bis 2015 (Lada 2017a: 16)

\begin{tabular}{|c|c|c|c|}
\hline Jahr & $\begin{array}{c}\text { Anzahl derjenigen, } \\
\text { die eine Fremdsprache } \\
\text { an der Schule lernen }\end{array}$ & $\begin{array}{c}\text { Anzahl derjenigen, } \\
\text { die Deutsch lernen }\end{array}$ & $\begin{array}{c}\text { Prozentanteil derjenigen, } \\
\text { die Deutsch lernen, } \\
\text { im Verhältnis zur Anzahl } \\
\text { derjenigen, die eine } \\
\text { Fremdsprache lernen }\end{array}$ \\
\hline 2010 & 8.188 .054 & 2.282 .822 & $27,9 \%$ \\
\hline 2011 & 7.778 .387 & 2.238 .187 & $28,8 \%$ \\
\hline 2012 & 7.528 .838 & 2.108 .790 & $28,0 \%$ \\
\hline 2013 & 7.705 .883 & 2.043 .904 & $26,5 \%$ \\
\hline 2014 & 8.017 .378 & 1.984 .742 & $24,8 \%$ \\
\hline 2015 & 8.245 .302 & 1.946 .523 & $23,6 \%$ \\
\hline
\end{tabular}

Auch das schwindende Interesse an der deutschen Sprache hat einen starken Einfluss auf die sinkenden Deutschlernerzahlen in den letzten Jahren. Der Untersuchung des Zentrums zur Erforschung der Öffentlichen Meinung (CBOS) aus dem Jahr 2015 zufolge liegt einer der Gründe dafür wohl in der Einstellung der meisten Lernenden und Studierenden, die angeben, dass eine Fremdsprache genüge, um kommunizieren zu können. Dabei geben 32\% der Probandinnen und Probanden Englisch, 20\% Russisch und 14\% Deutsch an (Lada 2017a: 12). Ein weiterer Grund für das geringere Interesse an der deutschen Sprache ist immer noch die negative Einstellung der Lernenden, die Deutsch mit einer schweren und unangenehm klingenden Sprache assoziieren. Es kommt häufig vor, dass die Schülerinnen und Schüler wegen des Mangels an alternativen Sprachlernangeboten mit dem Deutschlernen beginnen und keine Lust haben, ihre Sprachkenntnisse tatsächlich zu vertiefen, denn sie gehen davon aus, dass sie in den darauffolgenden Jahren höchstwahrscheinlich diese Sprache nicht gebrauchen werden (Ammon 2015: 1010; Mihułka 2016: 72-73, 77; Łada 2017a: 26).

Mit dem Rückgang des Interesses an der deutschen Sprache ließ in den letzten Jahren auch das Niveau des Deutschunterrichts nach. Laut Bericht des Auswärtigen Amtes (2015: 19) ist die Qualität der Deutschkenntnisse dort, wo Deutsch als zweite Fremdsprache gelernt wird, eher gering. In Anbetracht dessen, dass der polnische Rahmenlehrplan das Sprachniveau der Lernenden nach den jeweiligen Stufen im Gemeinsamen Europäischen Referenzrahmen für Sprachen bestimmt, lässt sich festhalten, dass das Sprachniveau A1 nach der Grundschule, A2 oder $\mathrm{A} 2+$ nach dem Gymnasium und B1 und B2 nach der Oberschule angestrebt werden sollte. Aus den durchgeführten Erhebungen ergibt sich allerdings, dass nur $10 \%$ der Gymnasialschülerinnen und -schüler das Niveau A2 erreichen. Der Anteil an Schülerinnen und Schülern, deren deutsche Sprachkenntnisse dem Niveau B1 und B2 entsprechen, beträgt 5 bis 7\% je nach der untersuchten Fertigkeit (Lada 2017a: 16). Das langsam sinkende Interesse an der deutschen Sprache ist auch bei 
der Wahl der deutschen Sprache als Abiturfach sichtbar, was der folgenden Tabelle zu entnehmen ist:

Tab. 2: Anzahl der Schülerinnen und Schüler sowie ihre Ergebnisse bei den Abiturprüfungen in Fremdsprachen im Schuljahr 2016/2017

\begin{tabular}{|l|c|c|c|c|c|c|}
\hline & \multicolumn{6}{|c|}{2017} \\
\cline { 2 - 7 } & Englisch & Deutsch & $\begin{array}{c}\text { Franzö- } \\
\text { sisch }\end{array}$ & Russisch & Spanisch & Italienisch \\
\hline Grundniveau & 237222 & 17493 & 752 & 4809 & 543 & 175 \\
\hline Erweitertes Niveau & 131839 & 7535 & 791 & 1983 & 671 & 341 \\
\hline $\begin{array}{l}\text { Durchschnittliches } \\
\text { Ergebnis - Grund- } \\
\text { niveau (\%) }\end{array}$ & 71 & 65 & 78 & 61 & 77 & 81 \\
\hline $\begin{array}{l}\text { Durchschnittliches } \\
\text { Ergebnis - erweit- } \\
\text { ertes Niveau (\%) }\end{array}$ & 60 & 56 & 61 & 57 & 55 & 68 \\
\hline
\end{tabular}

Anhand der in der Tabelle angeführten Daten sieht man, dass sich bis zu 59\% der Abiturienten für die Englischprüfung auf dem Grundniveau und 33\% für die Prüfung auf dem erweiterten Niveau entschieden. Die Anzahl der Abiturienten, die Deutsch als Abiturfach auf dem Grundniveau wählten, liegt bei ca. 4\%. Die Abiturprüfung in Deutsch auf dem erweiterten Niveau legten nur etwa 2\% der Lernenden ab. Wie die obige Tabelle zeigt, sind die Ergebnisse in Deutsch als Abiturfach neben Russisch am niedrigsten. ${ }^{4}$

Die Ursache für diese niedrigen Leistungen an polnischen Schulen könnte die oft geringe Wochenstundenzahl für die zweite Fremdsprache sein. Für den Deutschunterricht sind in der Regel nur zwei Wochenstunden vorgesehen (Auswärtiges Amt 2015: 19; Janoszczyk 2016: 55; Mihułka 2016: 71). Auch die Unterrichtsklassen sind mit meist über 20 Schülerinnen und Schülern viel zu groß, was die effiziente Entwicklung der fremdsprachlichen Fertigkeiten verhindert. Noch eine andere Ursache für die Vernachlässigung der DaF-Bildung liegt nach Łada (2017a: 25) im bisherigen Schulsystem. Sie stellt fest, dass die Lernenden sehr oft nach drei Schuljahren im Gymnasium auf den Deutschunterricht im weiterführenden Lyzeum verzichten. In den Oberschulen sind somit keine großen sprachlichen Fortschritte zu verzeichnen, weil sich die Schülerinnen und Schüler vor allem im letzten Schuljahr schon auf die Abiturfächer fokussieren. Einige fangen nach einem Schulwechsel mit dem Deutschunterricht von vorne an, was heißt, dass sie mit schwachen Sprachkenntnissen die Schule abschließen.

3 https://cke.gov.pl/egzamin-maturalny/egzamin-w-nowej-formule/wyniki/sprawozdanie-z-egzaminu-maturalnego-2017/ (Zugriff am 20.07.2018).

${ }^{4}$ Zur vergleichenden Analyse der Ergebnisse der schriftlichen Abiturprüfung in Deutsch und Englisch in den Jahren 2013, 2014, 2015 und 2016 siehe: Rolek 2016. 


\section{Zum gegenwärtigen Status der Hochschulgermanistik in Polen}

Der Studiengang Germanistik umfasst in Polen laut dem Bologna-Prozess drei Zyklen: ein sechssemestriges Bachelorstudium und ein darauf aufbauendes viersemestriges Masterstudium. Dem erfolgreichen Abschluss eines Masterstudiums folgt das Promotionsstudium mit eigenständiger Forschung und dem Erwerb eines Doktorgrades (Sieradzka 2016: 117-118; Sipowicz/Najbert/Radojewska 2016: 148; Żebrowska 2017: 103). Das Lehrangebot des Studiums beinhaltet obligatorische Lehrveranstaltungen, die sich vor allem auf Sprachpraxis, Literatur, Linguistik, beschreibende Grammatik und Landeskunde erstrecken.

Es gibt unterschiedliche Faktoren, die die Existenz der modernen Germanistik auf jeder dieser Bildungsstufen bedrohen können. Als das große Problem erweist sich jetzt der allmähliche Rückgang der Studierendenzahl. Seit 2005 gibt es keine Aufnahmeprüfung für das Germanistikstudium und die Immatrikulation erfolgt nur durch eine Rankingliste, die anhand der Ergebnisse der Reifeprüfung erstellt wird. Die Germanistik wird aber meistens nicht von Abiturienten mit den besten Ergebnissen gewählt, sondern von denjenigen, die sich für ein anderes Studium nicht qualifizierten: „Es wird etwa geschätzt, dass von 400 Germanistik-Kandidaten Personen vom zweihundertsten bis zum dreihundertsten Platz (der Reihenfolge der besten Abiturergebnisse folgend) immatrikuliert werden“ (Lada 2017a: 19). Aus anderen Schätzungen ist zu schlussfolgern, dass auch im Masterstudium immer weniger Studierende eingeschrieben sind. Nach drei Jahren schließen die meisten Germanistikstudierenden das Bachelorstudium ab und nur etwa ein Drittel entschließt sich dazu, das Masterstudium fortzusetzen. Die einen begründen ihre Wahl meistens damit, dass sie von dem Studienangebot enttäuscht sind. Die anderen geben offen zu, dass sie von vornherein planten, zunächst nur ihre Sprachkenntnisse zu verbessern und dann einen anderen Studiengang zu wählen, um die eigenen beruflichen Chancen zu vergrößern. Ein weiterer Grund für die niedrigere Studierendenzahl im Masterstudiengang liegt auch in der Schließung vieler Lehrerkollegs in den letzten Jahren (Lada 2017a: 19).

Obwohl Deutsch als Abiturfach als obligatorische Bedingung für die Aufnahme der Bewerberinnen und Bewerber ins Germanistikstudium gilt, sinkt das Niveau ihrer Deutschkenntnisse immer weiter ab (Szczęk/Kałasznik 2016: 106). ${ }^{5}$ Es kommt oft vor, dass immer mehr Studienanfängerinnen und -anfänger nicht über ausreichende Sprachkenntnisse verfügen, um an Lehrveranstaltungen, die auf Deutsch geführt werden, teilzunehmen. Kritisch beurteilt werden auch allgemeine

5 Zur Diagnose der Deutschkenntnisse der Kandidaten und Germanistikstudierenden an der Universität Wrocław siehe: Szczęk/Kałasznik 2016. 
Kompetenzen der Studierenden in den Wissensbereichen wie Literatur, Philosophie, Geschichte oder Geografie. Die Folge davon ist, dass diese Hochschulabsolventen letztendlich keine sehr guten Sprachkenntnisse aufweisen können: „Manche Hochschullehrer gehen in ihrer Einschätzung sogar soweit, dass das Sprachniveau der Hochschulabgänger in etwa dem Grad entspricht, wie er in den 1990er Jahren ausreichend war, um ein Germanistikstudium aufzunehmen“ (Łada 2017a: 38). Die sinkenden Sprachkenntnisse der Studierenden zu Studienbeginn sind auch ein Anstoß für einige Germanistiken in Polen, Bewerberinnen und Bewerber ohne Deutschkenntnisse (die sog. ,Nullanfänger") aufzunehmen. In diesem Zusammenhang wurde eine Umgestaltung der Studienpläne notwendig, infolge derer der Sprachunterricht und berufspraktische Fächer (wie z.B. Wirtschaftskommunikation, interkulturelle Kommunikation) in den Vordergrund rücken und die Relevanz der theoretischen Wissenschaft dabei immer geringer wird (Szczęk/Kałasznik 2016: 105; Żebrowska 2017: 104). Dies gilt auch für die Neuorientierung anderer, Lehrpläne bzw. Studiengänge. Vor dem Hintergrund der derzeitigen mangelnden Attraktivität des Lehrerberufs und des Rückgangs des Deutschlernens an den Schulen werden weitere neue Studienangebote eingeführt, die die Studierenden auf andere Berufe vorbereiten sollen. Als Beispiel dienen z.B. Universitäten in Poznań, Łódź oder Wrocław, wo Seminare für Business Deutsch angeboten werden, in denen „nicht Deutsch als Kultursprache, als Sprache der Dichter und Philosophen vermittelt wird, sondern Deutsch als Wirtschaftssprache“ (Żebrowska 2017: 104). Die Studierenden wollen nämlich immer häufiger konkrete, in der Praxis umsetzbare Fertigkeiten entwickeln, was jedoch dem Grundgedanken der universitären (Aus-) Bildung widerspricht:

Eine Fremdsprache im Studium zu erlernen, bedeutet nicht nur, in dieser Sprache kommunizieren zu können, sondern in ihr auch komplizierte (literarische, wissenschaftliche usw.) Texte lesen, aber auch verfassen zu können, sich einwandfrei zu jedem Thema äußern zu können, sich für die Kultur zu interessieren, kurz: in dieser Sprache denken zu können. Denn jede (natürliche) Sprache übernimmt nicht nur eine kommunikative, sondern auch eine kognitive Funktion. Ex definitione sollte die Philologie beides vereinen. (Żebrowska 2017: 104)

Ein weiterer Faktor, der die Existenz der Germanistik in Polen gefährden kann, ist der Mangel an einem ausreichend philologisch ausgebildeten Nachwuchs. Wegen der relativ niedrigen Besoldung der polnischen akademischen Welt entscheiden sich begabte junge Menschen immer seltener für eine wissenschaftliche Laufbahn. Grucza (2010: 1764-1765) erklärt diese Tendenz wie folgt: „Zwar war die Entlohnung von Akademikern auch vor der Wende sehr bescheiden, doch damals konnte man auch woanders nicht viel mehr verdienen. Jetzt können Absolventen der Germanistik außerhalb der Hochschulen besser besoldete Stellen finden“. 


\section{Zum Profil des Germanistikstudiums an der Schlesischen Universität Katowice}

Am Institut für Germanische Philologie der Schlesischen Universität Katowice werden aktuell unterschiedlich akzentuierte Studien angeboten:

- Deutsch (Lehramt) mit Schwedisch als zweiter Sprache,

- Ausbildung zum Fachübersetzer (Recht und Wirtschaft),

- Deutsche Sprache (ohne Vorkenntnisse): seit 2015/2016,

- Schwedische Sprache: seit 2015/2016.

Bis 2015/2016 gab es im Direktstudium noch eine Studienrichtung: „Kultur und Literatur der deutschsprachigen Länder“", aber wegen mangelnder Nachfrage besteht diese seither nicht mehr. Die Studiengänge, die im akademischen Jahr 2015/2016 eingeführt wurden, erfreuen sich zur Zeit großer Beliebtheit, dazu ein paar Daten aus dem 1. Studienjahr:

- Deutsche Sprache (ohne Vorkenntnisse) - 2015/2016: ca. 30 Studierende; 2016/2017: ca. 40 Studierende; 2017/2018: ca. 30 Studierende;

- Schwedische Sprache - 2015/2016: ca. 40 Studierende, 2016/2017: ca. 50 Studierende; 2017/2018: ca. 50 Studierende.

Die Anzahl der Personen, die die Studienrichtung: „Ausbildung zum Fachübersetzer“ wählen, ändert sich von Jahr zu Jahr, aber leider ist auch hier eine sinkende Tendenz erkennbar: Bis 2014/2015 gab es ca. 50 Studierende im 1. Studienjahr, 2015/2016: schon ca. 40 Studierende, 2016/2017 und 2017/2018: ca. 30 Studierende. Eine ähnliche Tendenz beobachten wir auch bei der Studienrichtung: „Deutsch (Lehramt) mit Schwedisch als zweiter Sprache“; dort gibt es im 1. Studienjahr nur 13 Studierende, ein bis zwei Jahre zuvor noch ca. 20-25 Studierende und vor 3-4 Jahren sogar noch ca. 30 Studierende.

Im Allgemeinen ist auch eine geringe Anzahl an Studierenden zu verzeichnen, die das Masterstudium als Direktstudium fortsetzen. Manche schließen das Bachelorstudium ab und nehmen das Masterstudium nicht mehr auf, weil sie zu arbeiten beginnen oder eine andere Fachrichtung wählen, um bessere Berufsperspektiven zu haben. Im Wochenendstudium im Masterstudiengang werden seit 2016/2017 bereits keine Kandidaten mehr aufgenommen. Von Jahr zu Jahr schrumpfte die Studierendenzahl: 2010/2011 gab es noch ca. 30-40 Studierende, vor zwei Jahren wurden nur 15 Studierende immatrikuliert.

\subsection{Sprachniveau der Germanistikstudierenden}

Um die sprachliche Kompetenzstufe C2 zu erlangen, nehmen die Studierenden am Germanistischen Institut der Schlesischen Universität Katowice am sprachpraktischen Unterricht teil. Laut Lehrplan umfasst der Sprachunterricht im ersten 
Studienjahr 240 Stunden, d.h.: kombinierte Fertigkeiten = Hörverstehen + Leseverstehen + Konversationen (120 Stunden), praktische Grammatik (60 Stunden), Phonetik (30 Stunden) und Schreiben (30 Stunden).

Um zu ermitteln, was die Studierenden tatsächlich nach dem Abitur schon können und was sie bislang noch nicht beherrschten, wird ein grammatisch-lexikalischer Einstufungstest am Institut für Germanische Philologie der Schlesischen Universität Katowice in der ersten Unterrichtswoche des jeweiligen akademischen Jahres durchgeführt. Der von dem Autor dieses Beitrags entwickelte Test beinhaltet sowohl Lücken- als auch Multiple-Choice-Übungen. Im grammatischen Teil, der sich aus sieben Übungen zusammensetzt, sollten die Studierenden die fehlenden Endungen der Artikel bzw. der Possessivpronomen einsetzen, die richtigen Verbformen ankreuzen, die Passivsätze den angegebenen Aktivsätzen zuordnen, die passenden Präpositionen und Konjunktionen markieren, Adjektivendungen ergänzen sowie Wunschsätze im Konjunktiv II bilden. Der lexikalische Teil umfasst dagegen drei Übungen: In einer Übung sind die fehlenden Wörter sinngemäß zu ergänzen, in einer anderen sollten die Studierenden die Wörter/Begriffe einsetzen, die aus den angegebenen Definitionen resultieren. Abschließend müssen sie einen kurzen Text durchlesen und jeweils ein konkretes Wort aus vier möglichen Antworten wählen, das in die Lücke passt.

Im Folgenden werden die Ergebnisse des Einstufungstests dargestellt und kommentiert, der im Oktober 2017 durchgeführt wurde. Zur Probandengruppe gehörten 55 Studierende des ersten Studienjahres (im Alter von 19-20 Jahren), die zum Lehrer und Fachübersetzer gezielt ausgebildet werden. In Anlehnung an das von dem Autor dieses Beitrags erstellte Lösungsblatt lässt sich die erreichte Punktzahl wie folgt veranschaulichen:

Tab. 3: Die von Studierenden erreichte Punktzahl im Einstufungstest

\begin{tabular}{|c|c|}
\hline Punktzahl & Studierendenzahl \\
\hline $11-20$ & 5 \\
\hline $21-30$ & 14 \\
\hline $31-40$ & 10 \\
\hline $41-50$ & 9 \\
\hline $51-60$ & 8 \\
\hline $61-70$ & 8 \\
\hline $71-80$ & 1 \\
\hline $81-90$ & 0 \\
\hline $91-100$ & 0 \\
\hline
\end{tabular}

Aus den hier aufgeführten Testergebnissen lässt sich ableiten, dass die meisten Probandinnen und Probanden schwache Sprachkenntnisse aufweisen. Zu beachten ist, dass alle getesteten Personen die Abiturprüfung in Deutsch bestanden, die zum 
Niveau B1 bzw. B2 hinführen sollte. In unserem Test erreichten sie aber nur zwischen $21 \%$ und $40 \%$ der Gesamtpunktzahl, was eher dem Niveau A2 entspricht.

Was den grammatischen Teil betrifft, bereitete den Studierenden die Übung große Schwierigkeiten, in der sie die Endungen der bestimmten und unbestimmten Artikel bzw. der Possessivpronomen im richtigen Kasus einsetzen sollten. 45\% der Probandinnen und Probanden ergänzten richtig nur die Hälfte der Lücken. Viele Probleme tauchten auch bei der Übung auf, wo die Adjektivendungen zu vervollständigen waren. Anhand der Ergebnisse lässt sich feststellen, dass $60 \%$ der Probandinnen und Probanden nur zwei von fünf Endungen korrekt einsetzen konnten. Der Grund dafür liegt höchstwahrscheinlich in der Unkenntnis der deutschen Artikel und Kasusformen. Ähnlich schlechte Ergebnisse wurden bei der Markierung der richtigen Verbformen erzielt. Rund 42\% der getesteten Personen kreuzten lediglich die Hälfte der angegebenen Formen richtig an, obwohl sie zum großen Teil mit sehr gängigen Verben aus dem Grundwortschatz wie z.B. geben, halten, schlafen, wissen, lesen im Präsens zu tun hatten. Das Beherrschen des Passivs, das mit Hilfe von 5 Sätzen überprüft wurde, fiel den Probandinnen und Probanden ebenfalls schwer. Ihre Aufgabe bestand darin, dass sie jeweils den Passivsatz anzukreuzen hatten, der dem angegebenen Aktivsatz entsprach. Sie mussten also keinen Satz umwandeln. Niemand markierte jedoch alle Antworten korrekt, nur 11 Studierende kreuzten vier richtige Antworten an, 18 - drei richtige Antworten, 15 - zwei richtige Antworten, 8 Personen - eine richtige Antwort und 3 Studierende kreuzten gar keine richtige Antwort an.

Aus der Analyse der gesammelten Angaben lässt sich zudem schließen, dass die Probandinnen und Probanden auch über eine geringe lexikalische Kompetenz verfügen. Rund 56\% der Studierenden lösten im Durchschnitt 20-30\% der Aufgaben korrekt. Sie kannten geläufige feste Wortverbindungen des Deutschen nicht, wie etwa: Erste Hilfe leisten, einen Ausflug machen, eine Entscheidung treffen. Die Defizite waren auch im Falle der alltäglichen Fragen und Reaktivformeln festzustellen. Es wurde u.a. ein Satz analysiert, der oft in Dialogen in den ersten Lehrwerkkapiteln auf Niveau A1 vorkommt und in dem die Lücke mit einem Fragewort zu ergänzen war: ,, sind Sie von Beruf? - Polizistin“. Dieser Satz wurde nur von 29 Studierenden (53\%) richtig vervollständigt, indem das Fragewort Was eingesetzt wurde. Weitere Antworten waren:

- Wer: 22 Studierende

- Wem oder mit wem: 2 Studierende

- Wie: 2 Studierende.

Außerdem fiel es den Probandinnen und Probanden schwer, dem Kontext bestimmte Informationen zu entnehmen. Bei der Übung, die in der Ergänzung von Begriffen anhand der vorgegebenen Definitionen bestand, trugen 38\% der Studierenden kein Wort ein. 24\% konnten nur einen einzigen Begriff richtig identifizieren. 


\subsection{Lernmotivation der Germanistikstudierenden}

Motivation ist ein komplexes Phänomen und besteht aus unterschiedlichen Komponenten, wie z.B. aus den allgemeinen individuellen Voraussetzungen, den Einstellungen zur Zielsprache, den Lernzielen, den bisherigen Erfahrungen und der Beurteilung der möglichen Leistung sowie den zielsprachenspezifischen Kontakten (Mackiewicz 2013: 206; Kenné 2015: 71; Riemer 2017: 11; Mericka 2017: 643).

In diesem Unterkapitel werden solche Faktoren ermittelt, die auf die Lernmotivation der Germanistikstudierenden einen entscheidenden Einfluss haben. Als Datenerhebungsinstrument wurden 55 Sprachlernbiografien herangezogen und ausgewertet, die von denselben Studierenden in der ersten Oktoberwoche schriftlich verfasst wurden, die auch an der im Unterkapitel 3.1. beschriebenen Messung der Sprachkompetenz beteiligt waren. Solche Sprachlernbiografien reflektieren vor allem „das Erleben von Sprachlichem“ (Franceschini 2002: 20), haben eine offene Form, d.h. sind längere oder kürzere narrative Texte über die Sprachlerngeschichte der Lernenden (Mackiewicz 2014: 207). Die Probandinnen und Probanden berichteten darin frei über ihre bisherigen Erfahrungen beim Lernen und Gebrauch des Deutschen als Fremdsprache und dabei vor allem über die damit verbundenen Motive und die (de)motivierenden Faktoren, ihre Ängste sowie Leistungen und Misserfolge im Deutschunterricht. Damit sich die Studienanfängerinnen und -anfänger in ihren Aussagen wegen der mehr oder weniger größeren Sprachbarrieren nicht eingeschränkt fühlten und somit die Qualität der gewonnenen Daten nicht beeinträchtigt wird, konnten sie ihre Sprachlernbiografien in ihrer Muttersprache schreiben (Mackiewicz 2014: 210; Mihułka 2016: 64; Riemer 2017: 11). ${ }^{6}$ Anhand der Aussagen, die den Sprachlernbiografien entnommen wurden, konnten folgende Aspekte identifiziert werden:

- Wissensmotiv,

- Nützlichkeitsmotiv,

- Erfolgsmotiv,

- Lehrermotiv,

- Abwechslung im Unterricht,

- Elternmotiv,

- Emotionen und Gefühle beim Deutschlernen.

Aus der Analyse der Sprachlernbiografien ergibt sich, dass die Germanistikstudierenden stark intrinsisch motiviert waren bzw. sind und aus eigenem Bedürfnis und Interesse Deutsch lernen wollen, was zur Entwicklung einer habituellen Motivation beiträgt. Wissensdrang und Neugier sind von dem zu lernenden Stoff angetrieben. Eine Studentin beschrieb beispielsweise, wie ihre Liebe zur deut-

${ }^{6}$ Ausgewählte Aussagen von Probandinnen und Probanden wurden vom Autor (M.J.) ins Deutsche übersetzt. 
schen Sprache durch Lieder entstand. Aus Neugier übersetzte sie sie, weil sie wissen wollte, worüber ihre Lieblingsbands konkret singen:

(1) Aus eigener Neugierde [...] griff ich nach dem Wörterbuch und „fischte“ die am meisten auftretenden Phrasen aus den Liedern „heraus“. Ich erinnere mich daran, dass dies auch der Grund dafür war, dass ich später aus meinem eigenen, inneren Bedürfnis ein Notizbuch führte, in das ich nützliche oder einfach meiner Meinung nach interessante und wohlklingende Wörter schrieb, die ich dann ins Polnische übersetzte.

Nach Meinung der Studierenden verstärkt die Bewältigung einer Aufgabe die Selbstverwirklichung und -entwicklung. Außerdem macht ihnen das Deutschlernen großen Spaß. Der stark motivierende Faktor ist eine Möglichkeit, die fremde Sprache in der Praxis, z.B. bei Begegnungen mit deutschen Muttersprachlern, zu verwenden:

(2) Als Gäste aus Deutschland zu unserem Gymnasium kamen, hatte ich die Gelegenheit, sie mit zwei Freundinnen zu begrüßen. Mein „Dialog“ basierte zwar nur auf den Worten: „Wir freuen uns, Sie kennen zu lernen“. Ich empfand dies jedoch als eine große Auszeichnung.

Was darüber hinaus am stärksten motivierend wirkt, sind nachweislich die eigenen Erfolgserlebnisse im Lernprozess. Eine Studentin erzählte z.B., wie sie einen Erfolg erlebte, indem sie ihren Freunden bei den Hausaufgaben half:

(3) Sowohl im Gymnasium als auch in der Oberschule half ich meinen Freundinnen in Deutsch. [...] Eine meiner Freundinnen sagte sogar (schon damals!), dass ich eine großartige Lehrerin wäre. Aus heutiger Sicht bewog mich vielleicht schon eine kleine Hilfe für andere dazu, das Lehramtsstudium aufzunehmen.

In diesem Fall war die Anerkennung der Lehrfähigkeiten der Studentin durch ihre Freundin ein Erfolg. Sie beobachtete, dass sie sich zum Lehren wirklich eignet und dass auch andere Personen es bemerken.

Sehr oft erleben Schülerinnen und Schüler auch dadurch sichtbare Erfolge, dass sie an verschiedenen Wettbewerben teilnehmen. Auf eine solche Art und Weise der Leistungsabfrage, die kompetitiv angelegt ist, wird ihre Motivation ebenfalls gesteigert:

(4) Nach einem Lernjahr gelang es mir, den 28. Platz im nationalen Deutschwettbewerb für Gymnasialschülerinnen und -schüler zu belegen. Dieses Ereignis wurde zweifellos zu einem der Hauptreize, das meine langfristige Motivation bestimmte.

Das alles führte dazu, dass sie sich für das Germanistikstudium entschied. Diese Motivation beeinflusst auch weiterhin die Lust auf die Verbesserung ihrer didaktischen Kenntnisse und Sprachfertigkeiten.

Ein weiterer und besonders ausgeprägter motivationaler Faktor ist die Lehrkraft, der eine wichtige Rolle in der Entwicklung der Lernmotivation sowohl bei Schülerinnen und Schülern als auch bei Studierenden zukommt. Ihre Vorgehensweise kann sowohl einen negativen als auch einen positiven Einfluss auf die Einstellung zum Deutschlernen haben. Unterstützung und Engagement der Lehrkraft 
sind im Fremdsprachenunterricht entscheidend und tragen zur positiven Einstellung der Lernenden zum Fremdsprachenlernen bei:

(5) Für mich war der Lehrerfaktor immer am wichtigsten. Er motivierte mich zum Lernen. So passierte es auch in diesem Fall. Trotz ihrer Strenge im Unterricht zog mich etwas zu dieser Lehrerin hin. Sie hielt die Gruppe mit einer starken Hand, aber im Unterricht konnte man immer auf ihre Hilfe zählen. Sie sagte nie: Ich habe keine Zeit, ich wiederhole nicht. Sie ärgerte sich nicht, wenn wir Fehler machten. [...] Sie hatte viel Geduld.

(6) In meinem Fall war das Lehrermotiv das wichtigste Motiv. [...] Ich wollte vor allem im Lehrer eine Person sehen, die mir hilft, mich unterstützt und mich nicht ständig kritisiert [...]. Meine Germanistin hob sich mit Engagement von anderen Lehrern ab, sie half immer und gab ihr Wissen sehr schnell und effektiv weiter. Ohne sie hätte ich in so kurzer Zeit nicht so viel erreicht. Die Persönlichkeit des Lehrers und sein Umgang mit Schülerinnen und Schülern können viel in der Einstellung zum Fach ändern.

Auch Qualifikationen und Kompetenzen der Lehrenden sind für die Lernergruppe von großer Bedeutung, was das weitere Zitat belegt:

(7) Ich hatte einen Deutschlehrer, der qualifiziert war und einen individuellen Umgang mit den Schülerinnen und Schülern hatte. Der Unterricht konzentrierte sich hauptsächlich auf die Kommunikation. Dadurch war es möglich, Sprachbarrieren zu überwinden und sich frei zu äußern. Die Schülerinnen und Schüler waren sehr aktiv und hatten keine Angst, Fehler zu machen.

Außerdem beeinflusste die abwechslungsreiche Unterrichtsgestaltung die Lernmotivation der Studienanfängerinnen und -anfänger stark. Eine Person hob z.B. hervor, dass die Einbettung von Musik in den Unterricht für sie sehr interessant war:

(8) Der Unterricht war mit anderen interessanten Arbeitsformen verbunden. Eine dieser Formen war beispielsweise der Einsatz von Musik im Deutschunterricht. Die Kombination von Lernen und Spaß war sehr interessant.

Auch andere Probandinnen und Probanden betonen den motivierenden Einfluss von Abwechslung im Unterricht:

(9) In der sechsten Klasse der Grundschule gab es einen Lehrerwechsel. Das war eine kompetente Person, die sich nicht nur auf das Lehren aus dem Lehrbuch konzentrierte. Sie organisierte verschiedene Sprachspiele, die die Schülerinnen und Schüler dazu ermutigten, weiter zu lernen. Im Rahmen von außerschulischen Aktivitäten organisierte sie zusammen mit den Schülerinnen und Schülern ein Puppentheater in der deutschen Sprache, das auf einer der Schulfeiern präsentiert, aufgenommen und zum Wettbewerb weitergeleitet wurde. [...] Sowohl damals als auch heute denke ich, dass das eine großartige Idee war. Das war sehr motivierend und ermöglichte das Lernen durch Spielen.

Die obige Aussage bestätigt nicht nur die motivierende Funktion eines abwechslungsreichen Unterrichts, sondern auch die wichtige Funktion des Dramas (auch in Form von Projektarbeit). Da in diesem Fall die Theateraufführung auch noch aufgenommen und für einen Wettbewerb vorgeschlagen wurde, empfanden es die Schülerinnen und Schüler als Bestätigung, dass sich ihre Anstrengung 
lohnte. Dieses Erfolgserlebnis motivierte zweifellos zur aktiven Teilnahme am Deutschunterricht.

An einem anderen Beispiel erkennt man, dass eine variierende Unterrichtsgestaltung starkes Interesse an der deutschen Sprache wecken kann:

(10) Die Lehrerin versuchte auch, unsere Aktivitäten durch Spiele, Gruppenarbeit, Lieder, Gedichte abwechslungsreich zu gestalten und führte sogar schrittweise die Unterrichtssprache ein. Der Unterricht gefiel mir immer besser und zu Hause benutzte ich das Internet und CDs zum Deutschlernen, um nach den deutschen Entsprechungen für die polnischen Wörter oder Ausdrücke zu suchen.

Die Aussage dieser Studentin verweist darauf, dass sie so intrinsisch motiviert war und ihr Wissen sogar zu Hause aus Eigeninitiative zu vertiefen begann. Zusammenfassend kann man sagen, dass auch hier die Lehrkraft einer der wichtigsten motivationalen Faktoren ist.

Die Lehrenden können allerdings die Lernmotivation auch negativ beeinflussen, wenn sie streng an der Realisierung des Lehrplans festhalten:

(11) Der Lehrer war nicht an Problemen interessiert, mit denen die Schülerinnen und Schüler konfrontiert wurden. Es war für ihn wichtig, das geplante Material umzusetzen. In der sechsten Klasse der Grundschule gab es einen Lehrerwechsel. Das war eine kompetente Person, die sich nicht nur auf das Lehren aus dem Lehrbuch konzentrierte.

(12) Die Lehrerin war sehr introvertiert, sogar melancholisch. Sie war oft im Unterricht nachdenklich, sie starrte nur in eine Richtung. Das dauerte drei Jahre lang [...]. Als die Lehrerin erfuhr, dass ich gerne die Abiturprüfung in Deutsch schreiben und dann Germanistik studieren wollte, riet sie mir davon ab und argumentierte, dass ich in der Schule keinen Job finde und mir das sparen sollte.

Ein anderes Beispiel gibt eine Studentin an, deren Englischlehrerin nicht hilfsbereit war und ihre Aufmerksamkeit nur auf die leistungsschwachen Schülerinnen und Schüler richtete. Ihre Vorgehensweise hatte zur Folge, dass sich diese Studentin für Deutsch zu interessieren begonnen hat.

Einen Einfluss auf die Lernmotivation der Studierenden hatten auch die Eltern. Eine Studentin beschrieb, dass ihre Eltern die ersten Personen waren, die ihre Liebe zur Fremdsprache entwickeln ließen. Sie kauften ihr unterschiedliche Bücher und Zeitschriften und dadurch ermunterten sie sie zum Lernen. Bei einer anderen Studentin trafen die Eltern die Entscheidung, dass sie ein paar Jahre in Deutschland wohnen würden. Diese Situation führte zur Entwicklung einer integrativen Motivation und zur Verbesserung der Deutschkenntnisse. Viele Studierende hatten auch dank ihrer Eltern zusätzliche Privatstunden, die ihnen beim Deutschlernen halfen.

Die Studierenden schrieben auch Einiges über die das Deutschlernen begleitenden Emotionen und Gefühle. $\mathrm{Zu}$ den positiven Emotionen gehören vor allem Stolz und Zufriedenheit mit dem erreichten Erfolg durch den Gewinn eines Sprachwettbewerbes, durch gute Benotung, beim Bestehen eines Tests oder einer Prüfung. Neben den positiven Gefühlen erlebten die Studierenden auch negative 
emotionale Zustände wie Stress und Angst. Die Probandinnen und Probanden hatten besonders Angst vor dem Sprechen, der Kritik, den zu hohen Anforderungen und der schlechten Benotung, was mit Gesichtsverlust und Versagensangst in der Gruppe verbunden war:

(13) Die Angst davor, dass ich von besseren Schülerinnen und Schülern verspottet werde, war enorm. Die Situation wurde vom Lehrer verschlechtert, der über die Schülerinnen und Schüler, ihre Mängel lachte und sie bei jedem Schritt kritisierte. Meine Angst vor dem Unterricht bewirkte, dass ich mich körperlich unwohl fühlte.

\section{Schlussbemerkungen}

Die hier durchgeführte Studie ermöglichte es, das Sprachniveau und die bisherigen Lernerfahrungen der Germanistikstudierenden der Schlesischen Universität Katowice zu analysieren und die sich daraus ergebenden Schlussfolgerungen zu evaluieren.

Aus den Daten, die anhand des durchgeführten Einstufungstests ermittelt wurden, ist zu schließen, dass die tatsächliche grammatische und lexikalische Kompetenz der Studierenden viel schwächer ist, als es anhand der Ergebnisse der Abiturprüfung in Deutsch zu vermuten wäre. Da die Hochschulgermanistik vom sinkenden Niveau der Sprachkenntnisse der aufgenommenen Studierenden immer häufiger betroffen ist, scheint es begründet zu sein, die Curricula für Deutsch als Fremdsprache im universitären Bereich so umzugestalten, dass ein immer stärkerer Fokus auf den sprachpraktischen Unterricht gelegt wird.

Von Bedeutung für unsere Untersuchung waren ebenfalls die Sprachlernbiografien, die es uns erlaubten, individuelle Perspektiven von Studierenden näher zu betrachten, obwohl sie wegen ihres subjektiven Charakters weder als repräsentativ gelten können noch generalisierbar sind. Sie enthalten jedoch wichtige Angaben, die Aufschluss über Motivation und damit verbundene Einfluss- und Begleitfaktoren geben.

Als ein entscheidender Faktor beim Deutschlernen erweist sich die Lehrkraft, die die Ausbildung einer starken Motivation im Lernprozess wesentlich beeinflusst: ,interessanter Unterricht [hat] generell große Chancen, motivierend zu wirken, wohl in erster Linie dann, wenn die Lehrperson ihr eigenes Interesse am Gegenstand und an seiner Vermittlung glaubhaft machen kann" (List 2002: 6). Die Studierenden unterstreichen vor allem die Bereitschaft ihrer Lehrerinnen und Lehrer (in der Schule), den Unterricht abwechslungsreich und zugleich durchaus herausfordernd zu gestalten. Solch eine Unterrichtsmethode wirkte sich darauf aus, dass die jungen Menschen das Germanistikstudium bewusst wählten und mit Neugier die Sprache und Kultur der deutschsprachigen Länder kennen lernen wollen.

Die Probandinnen und Probanden heben darüber hinaus die bedeutende Rolle der Gefühle und Emotionen im Fremdsprachenunterricht hervor. Die positiven 
Gefühle treten meistens dann auf, wenn die Studierenden eine Aufgabe erfolgreich bewältigen oder wenn ihre Anstrengungen bemerkt werden. Solche positiven Emotionen verstärken die Lernmotivation in besonderem Maße und erhöhen das Selbstwertgefühl der Lernenden. Beunruhigend ist allerdings das vermehrte Auftreten negativer Emotionen, die meistens mit Gesichtsverlust, Versagen sowie der Angst vor Fehlern und vor dem Umgang der Lehrkraft mit Fehlern verbunden ist. Aus diesem Grund dürfen die Lehrenden nicht vergessen, dass sie nicht nur ihre Pflichtaufgaben zu erfüllen haben, sondern auch die Mentorrolle erfüllen sollten, um das Interesse der Lernenden an der Sprache und dem Zielsprachenland zu wecken sowie sie zum Wissenserwerb zu ermuntern.

Es steht fest, dass das polnische Germanistikstudium in mancherlei Hinsicht einer Modernisierung bedarf. Nach wie vor widmet sich der Unterricht einzelnen historischen und literaturwissenschaftlichen Themen, an denen die Studierenden kein besonderes Interesse haben. Das aktuelle Studienangebot sollte deshalb gründlich überprüft werden, um den Erwartungen der Germanistikstudierenden angesichts des sich verändernden Arbeitsmarktes besser Rechnung zu tragen. Nach Meinung deutscher Unternehmer, ${ }^{7}$ die in Polen investieren und immer häufiger nach fachlich und sprachlich gut ausgebildeten Mitarbeiterinnen und Mitarbeitern aus verschiedenen Branchen suchen, beherrschen die polnischen Hochschulabsolventen leider oft nicht den deutschen Fachwortschatz. Sie sind auch nicht im Stande, in der Geschäftswelt (bei Telefongesprächen, Verhandlungen und Präsentationen) auf Deutsch angemessen zu kommunizieren. Dies liegt auch daran, dass solche Fähigkeiten in philologischen Studiengängen nicht gezielt vermittelt werden (Földes 2016: 15).

Damit die polnische Germanistik ihre derzeitige Position halten und vielleicht sogar stärken kann, ist beim Aufbau ihrer Lehr- und Forschungsprogramme die Tatsache zu beachten, dass sie ,in einem Land zu wirken hat, in dem sie vor allem von der Leistung des Deutschunterrichts an den Schulen des Landes abhängig ist" (Grucza 2010: 1765). Wenn keine Maßnahmen unternommen würden, besteht die Gefahr, dass die Anzahl der Studierenden immer mehr schrumpft, sodass weitere Studienrichtungen im Fach Germanistik von der Schließung bedroht sind.

${ }^{7}$ Für den Stellenwert der deutschen Sprache und der Germanistik sind die vielfältigen wirtschaftlichen, politischen und kulturellen Beziehungen zwischen Deutschland (und den anderen deutschsprachigen Staaten) und Polen von großem Belange (Ammon 2015: 1008; Janoszczyk 2016: 45): „Beispielsweise ist für Polen Deutschland der Handelspartner Nummer eins und für Deutschland belegte Polen 2015 beim Export den 8., beim Import den 6. Platz. In der deutsch-polnischen Industrie- und Handelskammer sind mehr als 1.000 Firmen registriert, es gibt 600 Städtepartnerschaften etc.“ (Földes 2016: 15) 


\section{Literatur}

Ammon, Ulrich (2015): Die Stellung der deutschen Sprache in der Welt. Berlin/München/Boston. Auswärtiges Amt (Hrsg.) (2015): Deutsch als Fremdsprache weltweit. Datenerhebung 2015. Berlin. Földes, Csaba (2016): Position und Entwicklungsdynamik des Deutschen als Fremdsprache international - im Blick der angewandten Linguistik. In: Glottodidactica. An International Journal of Applied Linguistics 43(2), S. 13-38.

Franceschini, Rita (2002): Sprachbiographien: Erzählungen über Mehrsprachigkeit und deren Erkenntnisinteresse für die Spracherwerbsforschung und die Neurobiologie der Mehrsprachigkeit. In: Bulletin suisse de linguistique appliquée 76, S. 19-33.

Grucza, Franciszek (2010): Deutsch in Polen. In: Krumm, Hans-Jürgen / Fandrych, Christian / Hufeisen, Britta / Riemer, Claudia (Hrsg.): Deutsch als Fremd- und Zweitsprache. Ein internationales Handbuch. 2. Halbband. Berlin/New York. S. 1761-1766.

Janoszczyk, Jolanta (2016): Deutsch - (immer noch?) die zweitpopulärste Fremdsprache in Polen. In: Mihułka, Krystyna / Sieradzka, Małgorzata / Budziak, Renata (Hrsg.): Die Fremdsprache Deutsch in Polen: Anfänge, Gegenwart, Perspektiven. Rzeszów. S. 43-60.

Kenné, Augustin (2015): Motivation und Demotivation im Deutschunterricht an der Universität Dschang. In: Info DaF 42(1), S. 71-86.

List, Gudula (2002): Motivation im Sprachunterricht. In: Fremdsprache Deutsch 26, S. 6-10.

Łada, Agnieszka (2017a): Floskeln oder Fakten? Zur Situation der deutschen Sprache in Polen. Fakten und Meinungen. Warszawa.

Łada, Agnieszka (2017b): Weltweit führend beim Deutschlernen. In: INFO - Magazin des Deutsch-Polnischen Jugendwerks 1, S. 4-5.

Mackiewicz, Maciej (2013): Motywacja i nastawienia polskich uczniów do nauki języka niemieckiego w kontekście różnic regionalnych i postrzegania społeczeństwa niemieckiego. In: Neofilolog. Czasopismo Polskiego Towarzystwa Neofilologicznego 40(2), S. 205-217.

Mackiewicz, Maciej (2014): Interkulturelle Motivation im Fremdsprachenunterricht. Eine komparative Studie zu Deutsch als Fremdsprache in Polen und den USA. Frankfurt am Main u.a.

Mericka, Kirsten (2017): Motive für das Lernen von Deutsch als Fremdsprache am Wellesley College (USA) und an der Universidade Federal de Minas Gerais (Brasilien). In: Info DaF 44(5), S. 641-659.

Mihułka, Krystyna (2016): Der Deutschunterricht in Polen - Bemerkungen der Germanistikstudenten der Universität Rzeszów nach dem Abschluss des pädagogisch-didaktischen Praktikums. In: Mihułka, Krystyna / Sieradzka, Małgorzata / Budziak, Renata (Hrsg.): Die Fremdsprache Deutsch in Polen: Anfänge, Gegenwart, Perspektiven. Rzeszów. S. 61-82.

Riemer, Claudia (2017): Was Deutschlernende berichten und was wir daraus über Motivation und Motivierung lernen können. In: Fremdsprache Deutsch 57, S. 10-14.

Rolek, Bogusława (2016): Die DaF-Abiturprüfung in Polen. Versuch einer synthetischen Darstellung. In: Mihułka, Krystyna / Sieradzka, Małgorzata / Budziak, Renata (Hrsg.): Die Fremdsprache Deutsch in Polen: Anfänge, Gegenwart, Perspektiven. Rzeszów. S. 83-98.

Sieradzka, Małgorzata (2016): Sprachpraktische Ausbildung in der Struktur des Germanistikstudiums nach dem Bologna-Prozess am Beispiel des BA-Studiengangs im Institut für Germanistik an der Universität Rzeszów. In: Mihułka, Krystyna / Sieradzka, Małgorzata / Budziak, Renata (Hrsg.): Die Fremdsprache Deutsch in Polen: Anfänge, Gegenwart, Perspektiven. Rzeszów. S. 117-131.

Sipowicz, Kasper / Najbert, Edyta / Radojewska, Aleksandra (2016): Germanistik in den USA und Polen. Versuch einer komparatistischen Analyse. In: Żebrowska, Ewa / Olpińska-Szkiełko, Magdalena / Latkowska, Magdalena (Hrsg.): Beiträge zur Germanistik. Zwischen Kontinuität und Modernität. Metawissenschaftliche und wissenschaftliche Erkenntnisse der germanistischen Forschung in Polen. Warszawa. S. 142-155.

Germanica Wratislaviensia 144, 2019

(C) for this edition by CNS 
Szczęk, Joanna / Kałasznik, Marcelina (2016): Deutsch als Fremdsprache im universitären Bereich - Diagnose und Perspektiven für die Zukunft. In: Mihułka, Krystyna / Sieradzka, Małgorzata / Budziak, Renata (Hrsg.): Die Fremdsprache Deutsch in Polen: Anfänge, Gegenwart, Perspektiven. Rzeszów. S. 99-116.

Żebrowska, Ewa (2017): Deutsch und Germanistik in Polen. In: Mitteilungen des Deutschen Germanistenverbandes 64, Themenheft: Germanistik in Europa: Forschungs- und Ausbildungsperspektiven, S. 101-105.

https:/cke.gov.pl/egzamin-maturalny/egzamin-w-nowej-formule/wyniki/sprawozdanie-z-egzaminu-maturalnego-2017/ (Zugriff am 20.07.2018). 